\title{
iTRAQ-based quantitative proteomic analysis of cerebrospinal fluid reveals NELL2 as a potential diagnostic biomarker of tuberculous meningitis
}

\author{
YONGTAO YANG ${ }^{1-3^{*}}, \mathrm{JUN}^{1-3^{*}}$, GUANGHUI CHEN ${ }^{2,3^{*}}$, YUAN ZHAN $^{2,3^{*}}$, JIAJU ZHONG $^{2-4}$, \\ YOUDONG WEI ${ }^{1-3}, \mathrm{KE} \mathrm{CHENG}^{1-3}, \mathrm{BIN}^{\mathrm{Q}} \mathrm{CIN}^{1-3}, \mathrm{HONGMIN} \mathrm{YOU}^{2,3}$ and PENG XIE ${ }^{1-3}$ \\ ${ }^{1}$ Department of Neurology, The First Affiliated Hospital of Chongqing Medical University; \\ ${ }^{2}$ Institute of Neuroscience, Chongqing Medical University; ${ }^{3}$ Chongqing Key Laboratory of Neurobiology; \\ ${ }^{4}$ Department of Neurology, Yongchuan Hospital of Chongqing Medical University, Chongqing, P.R. China
}

Received June 9, 2014; Accepted February 17, 2015

DOI: $10.3892 /$ ijmm.2015.2131

\begin{abstract}
Tuberculous meningitis (TBM) is a serious complication of tuberculosis that affects the central nervous system. As TBM may result in permanent sequelae and death, rapid, accurate diagnostic tests using novel biomarkers are required for the early diagnosis and treatment of TBM. A quantitative proteomic study was therefore performed to identify differential proteins in the cerebrospinal fluid (CSF) obtained from TBM patients $(n=12)$ and healthy controls $(n=12)$. CSF samples were labelled with iTR AQ ${ }^{\mathrm{TM}}$ and analyzed by LC-MS/MS. Gene Ontology and Pathway Analysis were conducted using DAVID bioinformatics resources. Neural epidermal growth factor-like like 2 (NELL2) with the largest fold-change value was selected for validation by western blotting. Proteomic phenotyping revealed over-representation in two inflammation-associated processes, complement and coagulation cascades as well as cell adhesion molecules. Western blotting showed a significant decrease in NELL2 levels in TBM subjects compared to healthy controls. The AUC analysis revealed NELL2 was able to distinguish TBM subjects from healthy controls with $83.3 \%$ sensitivity and $75 \%$ specificity. In conclusion, the results showed that CSF NELL2 is a potential diagnostic biomarker for TBM. Further evaluation of these findings in larger studies including anti-tuberculosis medicated and unmedicated patient cohorts with other intracranial infectious diseases is required for clinical translation.
\end{abstract}

Correspondence to: Professor Peng Xie, Department of Neurology, The First Affiliated Hospital of Chongqing Medical University, 1 Yixue Road, Yuzhong District, Chongqing 400016, P.R. China

E-mail: xiepeng@cqmu.edu.cn

${ }^{*}$ Contributed equally

Key words: tuberculous meningitis, cerebrospinal fluid, biomarker, iTRAQ, proteomic

\section{Introduction}

Each year, almost 10 million individuals worldwide are newly diagnosed with tuberculosis (TB), an infectious disease caused by Mycobacterium tuberculosis (MTB) with 1.7 million mortalities owing to TB (1). Moreover, two billion individuals worldwide with latent MTB infection represent a potential risk of TB reactivation (2). The burden of disease of TB is highest in Asia, with China listed only second to India. In the latest Global Tuberculosis Report by the World Health Organization (WHO), China accounts for $12 \%$ of the TB cases worldwide. According to the National Survey of Drug-Resistant TB conducted by the Chinese Center for Disease Control, China also has a serious epidemic of drug-resistant TB (3), with 5.7\% of newly diagnosed cases and $25.6 \%$ of previously treated cases being multidrug-resistant (MDR) TB.

TB affects the central nervous system (CNS); a condition termed CNS tuberculosis, and is the most severe complication of TB infection. Tuberculous meningitis (TBM) is the most common form of CNS tuberculosis. In TBM patients, MTB in Rich foci (previously seeded and formed in the meninges or brain parenchyma through primary infection) reactivate and rupture into the subarachnoid space, producing meningitis. As TBM may result in permanent sequelae and death, prompt diagnosis and treatment are required (4). Prognosis depends on the clinical stage at which TBM is diagnosed (5). Effective TBM control requires early, efficient, and accurate diagnostic biomarkers in addition to drug-resistance identification and treatment response evaluation (6-8). However, the currrent gold standard for TBM diagnosis, MTB isolation from cerebrospinal fluid (CSF) culture, usually takes weeks to reach a positive outcome and has a relatively low sensitivity of $25-70 \%$ (9).

Since these conventional methods do not satisfy the requirements for diagnosis of TBM, it is crucial to target complementary disease-specific biomarkers that can facilitate earlier diagnosis and treatment in TBM patients. However, most studies on TB biomarkers have focused on pulmonary TB. In previous studies it was suggested that diagnostic biomarkers for TBM, were adenosine deaminase isoenzyme-2 
(ADA2) (10), adenosine deaminase (ADA) (11,12), 65-kDa heat shock protein antigen (13), CSF lactate, CSF lactate dehydrogenase (14) and arachidonate 5-lipoxygenase (15). However, none of these biomarkers have been sufficiently validated (16).

Therefore, rapid, accurate diagnostic tests using novel biomarkers for TBM remain to be developed. To that end, mass spectrometry (MS)-based quantitative proteomics has become a useful tool in identifying disease-specific biomarkers (17) by yielding information about differences across a spectrum of proteins within samples in a single experiment. Kumar et al (18) have applied an MS-based quantitative proteomic approach to compare postmortem brain tissues from confirmed cases of TBM with uninfected brain tissues and found upregulated levels of signal-regulatory protein $\alpha$ (SIRPA), protein disulfide isomerase family A, member 6 (PDIA6), amphiphysin (AMPH) and neurofascin (NFASC) in addition to downregulated levels of ferritin light chain (FTL). Although all the postmortem brain tissue samples were collected within 8-16 $\mathrm{h}$ of death with the patients' bodies maintained at $4^{\circ} \mathrm{C}$, proteins are rapidly degraded after death. Thus, there is a reasonable risk that TBM-specific proteins may have been missing or severely altered in the study of Kumar et al. As opposed to brain tissue, CSF can more easily be collected from live patients by lumbar puncture. As the CSF is in constant exchange with the brain's interstitial fluid and thus alterations in protein expression within the brain may be closely reflected in the CSF $(17,19)$, the CSF appears to be a more promising source of biomarkers for TBM. Among MS-based approaches, the isobaric tag for relative and absolute quantitation (iTR $\mathrm{AQ}^{\mathrm{TM}}$ )-based proteomics has emerged as particularly valuable in detecting relatively scarce proteins, such as those in the $\operatorname{CSF}(18,20)$.

In the present study, an iTRAQ ${ }^{\mathrm{TM}}$-based quantitative proteomic approach was used to identify differential proteins in the CSF obtained from live TBM patients and healthy controls. Proteomic phenotyping of the 81 differential proteins revealed over-representation in two inflammation-associated processes, complement and coagulation cascades as well as cell adhesion molecules. Western blotting of the neuron-specific differential protein with the largest absolute fold-change value, neural epidermal growth factor-like like 2 (NELL2), showed significantly decreased NELL2 levels in TBM subjects compared to healthy controls. Area under the receiver operating characteristic curve analysis demonstrated NELL2 was able to distinguish TBM subjects from healthy controls with $83.3 \%$ sensitivity and $75 \%$ specificity, indicating that CSF NELL2 shows promise as a diagnostic biomarker for TBM.

\section{Materials and methods}

Subjects. The protocol of the present study and the procedures employed for sample collection were approved by the Ethics Committee of Chongqing Medical University and have therefore been performed in accordance with the ethical standards laid down in the 1964 Declaration of Helsinki and its later amendments. Written informed consent was obtained from all participants. Twelve TBM subjects were diagnosed following the standard criteria (21) and enrolled in the Department of Neurology at the First Affiliated Hospital of Chongqing Medical University. Twelve healthy control (HC) subjects were enrolled after written informed consent was obtained in the medical examination center at the First Affiliated Hospital of Chongqing Medical University. The HC subjects were required to have neither active TB nor any neurological complaints.

Protein digestion and $i T R A Q^{T M}$ labeling. Pooled CSF samples were generated by combining equal volumes of the 12 individual plasma samples from each group. Each sample $(5 \mathrm{ml})$ was desalted and concentrated to $80 \mathrm{ml}$ in a Vivaspin spin column with a molecular weight cut-off of $5 \mathrm{kDa}$ (Vivascience AG, Hannover, Germany). Then, $120 \mu \mathrm{g}$ of protein from each sample was dissolved in $30 \mu \mathrm{l}$ of STD buffer (4\% SDS, $100 \mathrm{mM}$ DTT, $150 \mathrm{mM}$ Tris- $\mathrm{HCl}$ $\mathrm{pH} 8.0$ ), boiled in water for $5 \mathrm{~min}$, cooled to room temperature, diluted with $200 \mu \mathrm{l}$ of UA buffer ( $8 \mathrm{M}$ Urea, $150 \mathrm{mM}$ Tris- $\mathrm{HCl} \mathrm{pH} 8.0$ ), and transferred to $30 \mathrm{kDa}$ ultrafiltration. The samples were centrifuged at $14,000 \mathrm{x}$ g for $15 \mathrm{~min}$ and $200 \mu$ of UA buffer was added. The samples were centrifuged again for $15 \mathrm{~min}$ under the same conditions. Then, $100 \mu \mathrm{l}$ of $0.05 \mathrm{M}$ iodoacetamide in UA buffer was added, and the samples were incubated for $20 \mathrm{~min}$ in the dark. After $10 \mathrm{~min}$ of centrifugation at the above conditions, the filters were washed three times with $100 \mu \mathrm{l}$ of UA buffer. Then, $100 \mu \mathrm{l}$ of DS buffer (50 mM triethylammoniumbicarbonate, $\mathrm{pH} 8.5$ ) was added to the filters, and the samples were centrifuged for $10 \mathrm{~min}$ at the above conditions. This step was repeated twice. Subsequently, $2 \mu \mathrm{g}$ of trypsin (Promega, Madison, WI, USA) in $40 \mu 1$ of DS buffer was added to each filter, and the samples were incubated overnight at $37^{\circ} \mathrm{C}$. The resulting peptides were collected by centrifugation. The filters were rinsed with $40 \mu \mathrm{l}$ of $10 \mathrm{X}$ DS buffer again. $\mathrm{TRA}^{\mathrm{TM}}$ labeling was performed according to the manufacturer's instructions (Applied Biosystems, Foster City, CA, USA). As each sample consisted of a pool of CSF from 12 TBM subjects or $12 \mathrm{HC}$ subjects, iTRAQ ${ }^{\text {TM }}$ reagents 114 and 116 were applied to the HC sample and reagents 115 and 117 were applied to the TBM sample. The analytic processes, including plasma depletion, protein digestion, iTRAQ ${ }^{\mathrm{TM}}$ labeling, SCX fractionation and LC-MS/MS analysis, were repeated twice.

Peptide fractionation with strong cation exchange chromatography. Prior to liquid chromatography coupled with tandem mass spectrometry (LC-MS/MS) analysis, the peptides were purified from excess labeling reagent by strong cation exchange (SCX) chromatography. Briefly, the peptides were dried in a vacuum concentrator, dissolved in strong cation exchange buffer $\mathrm{A}\left(10 \mathrm{mM} \mathrm{KH}_{2} \mathrm{PO}_{4}\right.$ in $25 \%$ of $\left.\mathrm{ACN}, \mathrm{pH} 3.0\right)$, and loaded onto a Polysulfoethyl $4.6 \times 100 \mathrm{~mm}$ column $(5 \mu \mathrm{m}$, $200 \AA$ A PolyLC Inc., Columbia, MD, USA) at a flow rate of $1 \mathrm{ml} / \mathrm{min}$. A suitable gradient elution was applied to separate peptides at a flow rate of $1 \mathrm{ml} / \mathrm{min}$ with elution buffer $(10 \mathrm{mM}$ $\mathrm{KH}_{2} \mathrm{PO}_{4}, 500 \mathrm{mM} \mathrm{KCl}$ in $25 \%$ acetonitrile, $\mathrm{pH}$ 3.0). Eluted peptides were collected and desalted by an offline fraction collector. The resulting fractions were combined to 10 pools and desalted on C18 Cartridges [Empore ${ }^{\mathrm{TM}}$ SPE Cartridges C18 (standard density), bed I.D. $7 \mathrm{~mm}$, volume $3 \mathrm{ml}$; Sigma, St. Louis, MO, USA]. Each final fraction was concentrated by a vacuum concentrator and resuspended in $40 \mu \mathrm{l}$ of $0.1 \%(\mathrm{v} / \mathrm{v})$ trifluoroacetic acid. All the samples were stored at $-80^{\circ} \mathrm{C}$ until subsequent LC-MS/MS analysis. 
$L C-M S / M S$ analysis. For each fraction, $10 \mu 1$ of solution was injected for nano LC-MS/MS analysis using an AB SCIEX TripleTOF 5600 MS (Toronto, Concord, ON, Canada) equipped with a splitless Eksigent nanoUltra 2D Plus nanoLC system and a cHiPLC Nanoflex microchip system (Eksigent, Dublin, CA, USA). The Nanoflex system uses replaceable microfluidic traps and columns packed with ChromXP C18 (3 $\mu \mathrm{m}, 120$ A) for online trapping, desalting and analytical separations. The sample was loaded, and trapping and desalting were performed at $2 \mu \mathrm{l} / \mathrm{min}$ for $10 \mathrm{~min}$ with $100 \%$ mobile phase A (2\% acetonitrile/ $0.2 \%$ formic acid/ $98 \%$ water). For peptide elution, the gradient was initiated at $5 \%$ mobile phase $\mathrm{B}$ (98\% acetonitrile/ $0.2 \%$ formic acid/2\% water) and increased to $24 \%$ linearly in $70 \mathrm{~min}$ at a flow rate of $300 \mathrm{nl} / \mathrm{min}$. The total gradient length was $120 \mathrm{~min}$. MS data acquisition was performed in the information-dependent acquisition (IDA) mode. For TOF MS scans, the TripleTOF 5600 MS was operated with a resolving power of 30,000 FWHM. IDA survey scans were acquired in $250 \mathrm{msec}$ with a mass range of $\mathrm{m} / \mathrm{z}$ 350-1250. Specifically, 30x100 msec MS/MS were followed at a $120 \mathrm{cps}$ (counts/sec) trigger with a precursor charge state of +2 to +5 . Dynamic exclusion was set for $18 \mathrm{sec}$. Collision energies were calculated on-the-fly for all precursor ions using empirical equations based on mass and charge, and the Enhance iTRAQ ${ }^{\mathrm{TM}}$ function was turned on to improve the efficiency of the collision-induced dissociation.

Protein identification and quantification. All LC-MS/MS data were processed using ProteinPilot v4.2 and used to search the Uniprot_HUMAN.20140108_134997 database using the 'thorough search' option. The 'iTRAQ ${ }^{\mathrm{TM}}$ 8-plex peptide labeled' sample type and a 'biological modification ID focus' were selected for the analysis method. Trypsin was selected as the digestion enzyme, with cysteine alkylation by iodoacetamide as a modification. Data were normalized by ProteinPilot. The reported data were based on a false discovery rate (FDR) $\leq 1 \%$ confidence for protein identification. Differential proteins were analyzed by ProteinPilot for significant downregulation or upregulation. A significance threshold of $95 \%$ was selected as a criterion for each individual experiment. Peptides that matched multiple proteins were not included in the software quantification. The final ratios were calculated from the average of the ratios obtained in the two independent experiments in which the protein abundance was significant $(\mathrm{P}<0.05)$. In each experiment, bias correction for unequal mixing in the differently labeled samples was performed based on the assumption that most proteins do not change in expression. Thus, if the sample from each experimental condition was not combined in exactly equal amounts, this bias correction would fix the systematic error. The software identifies the median average protein ratio and corrects it to unity, and then applies this factor to all quantification results.

Gene Ontology and Pathway Analysis. Functional clustering was conducted using DAVID Bioinformatics Resources v6.7 (http://david.abcc.ncifcrf.gov/home.jsp) (22). A functional annotation analysis was performed with the Gene Ontology tool (GOTERM_CC_ALL) using the UniProt accession numbers for all the differential proteins. Only those GO terms in each gene list that yielded a P-value of $<0.05$ using
Table I. Demographic and clinical characteristics of the participants.

\begin{tabular}{lccc}
\hline Variables & HC & TBM & $\begin{array}{c}\text { P-value } \\
\text { (HC vs. TBM) }\end{array}$ \\
\hline Sample size & 12 & 12 & \\
Gender (M/F) & $7 / 5$ & $5 / 7$ & 0.44 \\
Age (years) & $27.7 \pm 5.3$ & $28.3 \pm 8.2$ & 0.09 \\
BMI $^{\mathrm{a}}$ & $46.1 \pm 10.0$ & $36.9 \pm 14.6$ & 0.85 \\
TB culture $_{\text {Fungal culture }}$ & $12 / 12$ & $0 / 12$ & - \\
\hline
\end{tabular}

HC, healthy controls;TBM, patients infected with tuberculous meningitis; M, male; F, female; BMI, body mass index; TB, tuberculosis. ${ }^{\mathrm{a}} \mathrm{Age}$ and $\mathrm{BMI}$ are presented as the means $\pm \mathrm{SD}$.

Fisher's exact test were considered significantly enriched. A Kyoto Encyclopedia of Genes and Genomes (KEGG) pathway analysis was subsequently performed using the same software, in which a corrected P-value of $<0.05$ was deemed to indicate a statistically significant difference.

Western blotting validation. Western blotting was performed on the 12 individual TBM samples and 12 individual HC samples. CSF samples were separated by $10 \%$ SDS-PAGE, blocked in 5\% skim milk in TBST, and transferred onto PVDF membranes. The membranes were incubated overnight at $4^{\circ} \mathrm{C}$ with an anti-NELL2 (diluted 1:1,000; Abcam, Cambridge, MA, USA) primary antibody. The membranes were washed and incubated with goat polyclonal to rabbit IgG horseradish peroxidase-coupled secondary antibody (diluted 1:10,000; Bio-Rad Laboratories, Hercules, CA, USA). After extensive washing, the protein bands detected by the antibodies were visualized by the enhanced chemiluminescence method and exposed to autoradiography film. After immunodetection, the membranes were stained with Ponceau S as an internal control for normalization. The western blot signals were densitometrically quantified in each sample with Quantity One (Bio-Rad Laboratories).

Statistical analysis. Statistical analysis was performed using the Statistical Package of Social Science (SPSS) for Windows v19.0. Data are expressed as the means \pm SD. The Student's t-test was applied to identify proteins with significant abundance differences between the TBM and HC subjects. Areas under the receiver operating characteristic (ROC) curve (AUC) were calculated to evaluate classifier performance. All the tests were two-tailed. The significance threshold was set at $\mathrm{P}<0.05$.

\section{Results}

Subject characteristics. Demographic and clinical data obtained from TBM and HC subjects are provided in Table I. The two groups of subjects were not differentiable by any key demographic characteristics, such as age, gender or body mass index (BMI). 


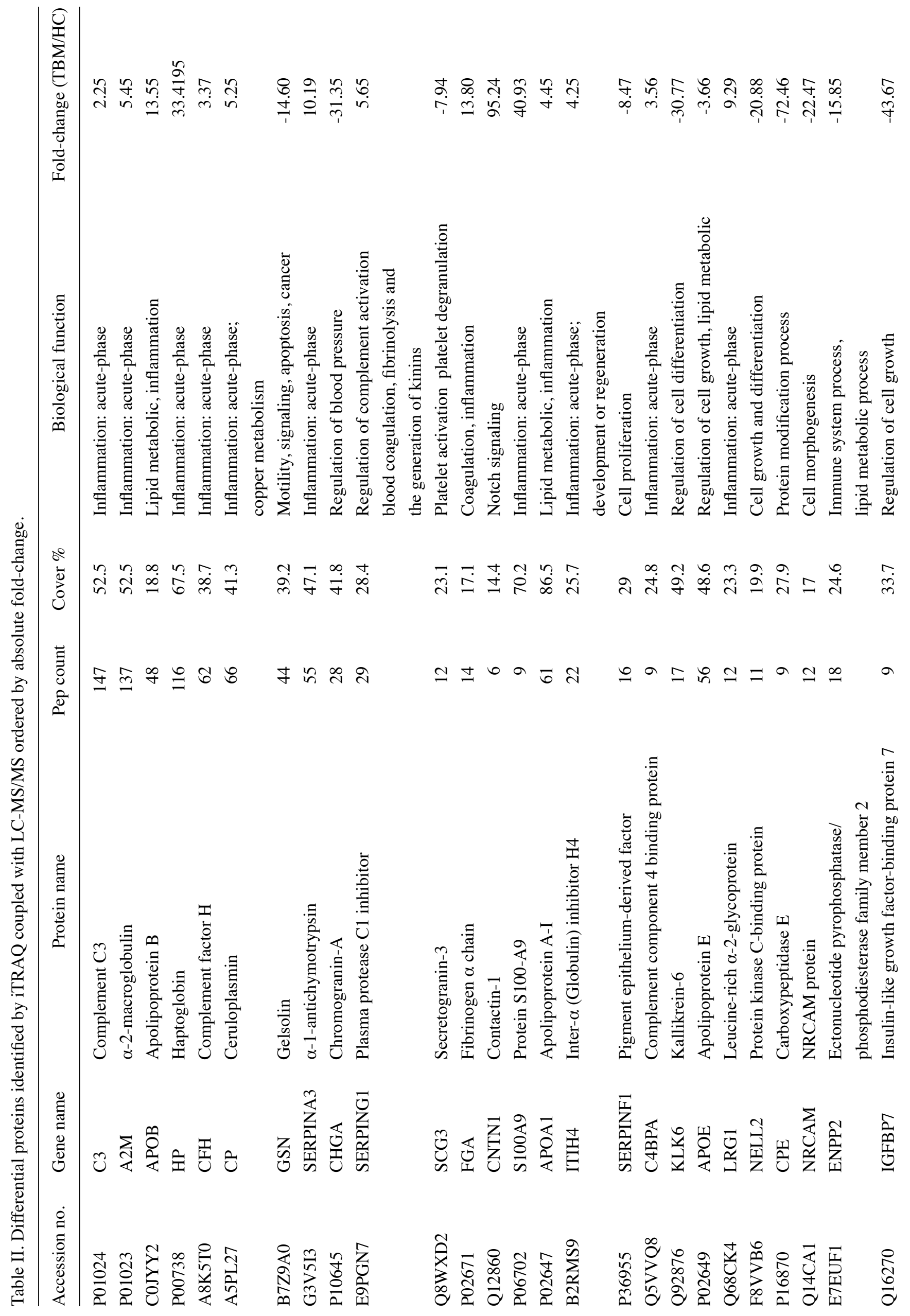




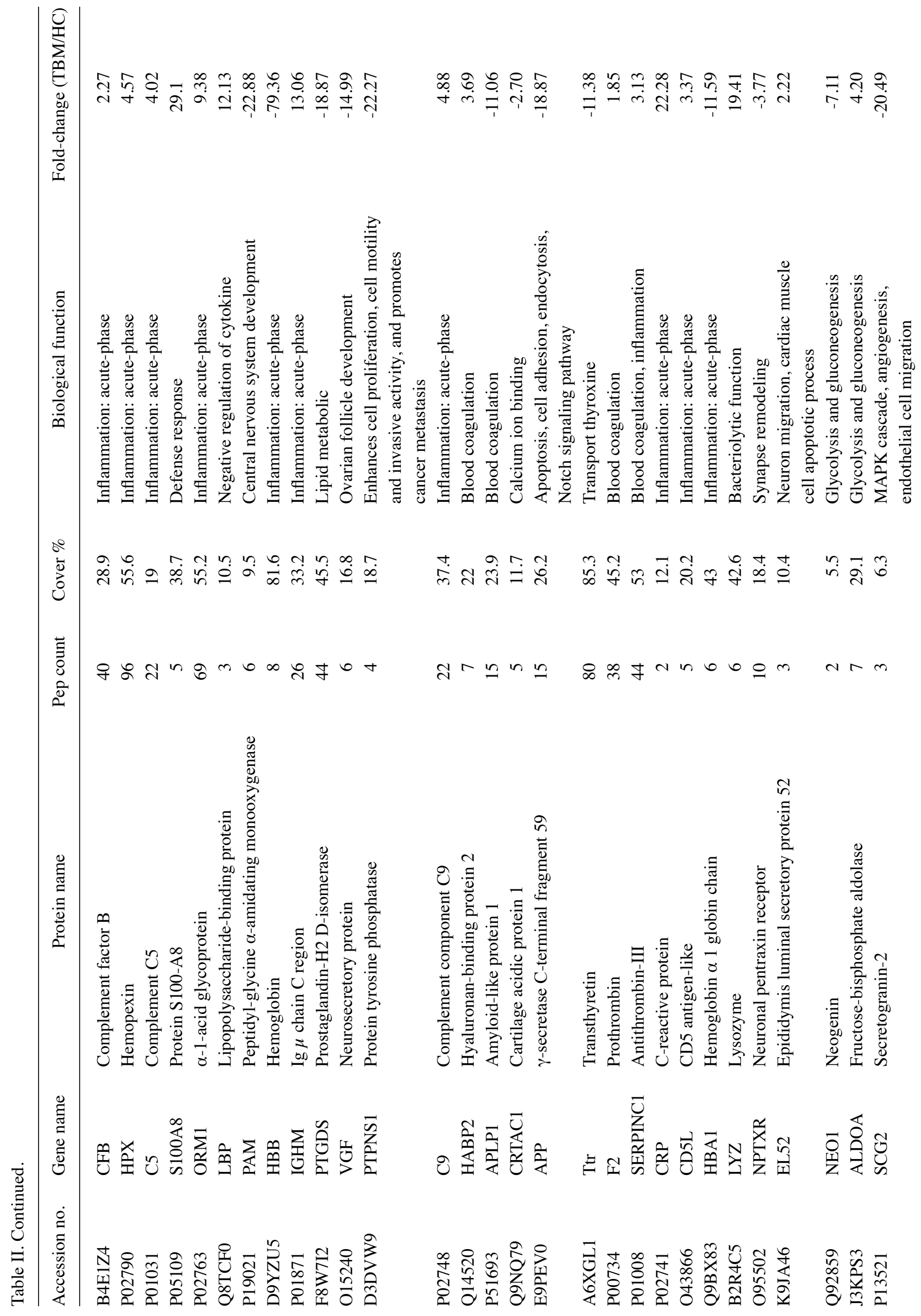




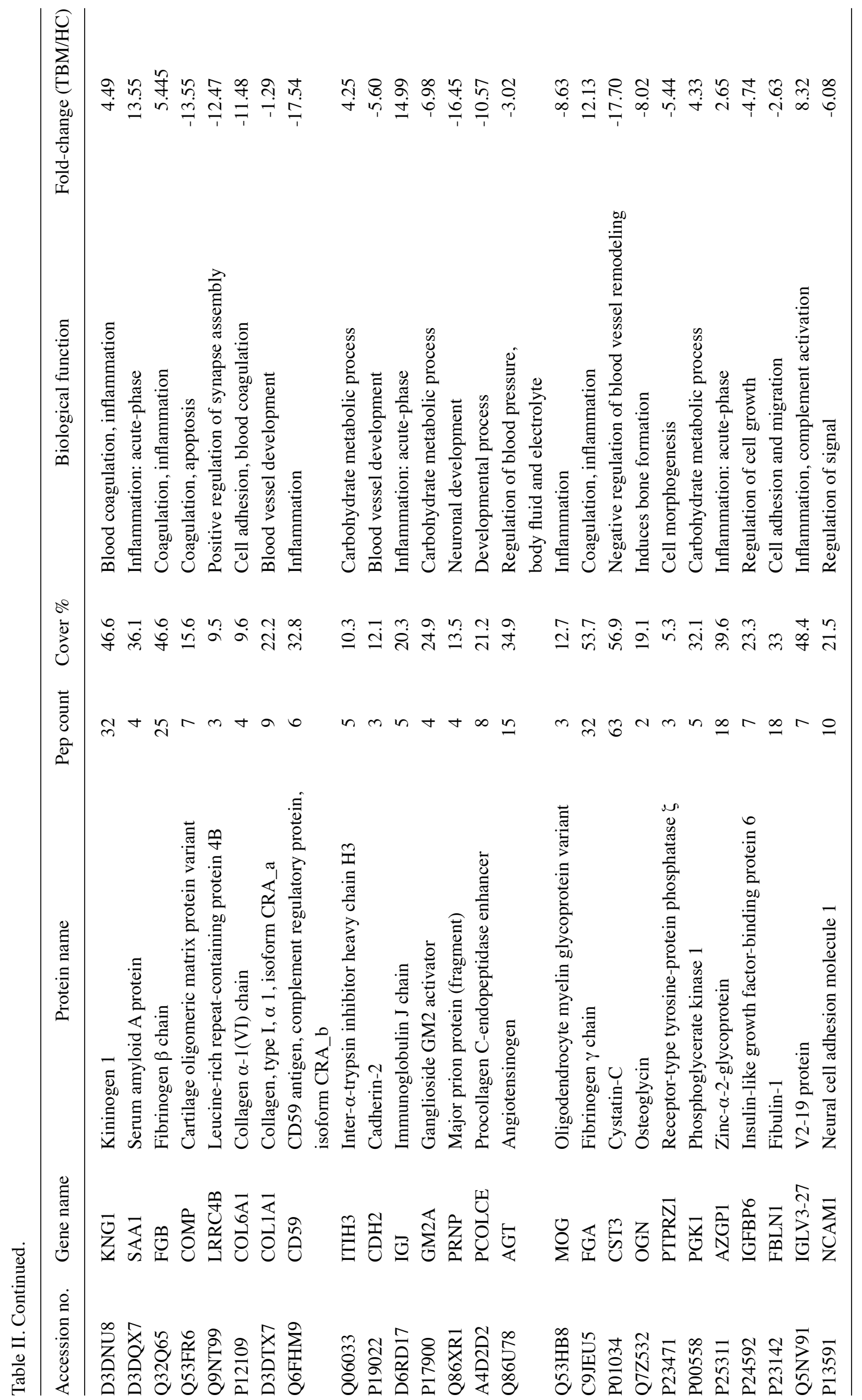




\section{A Biological Process}

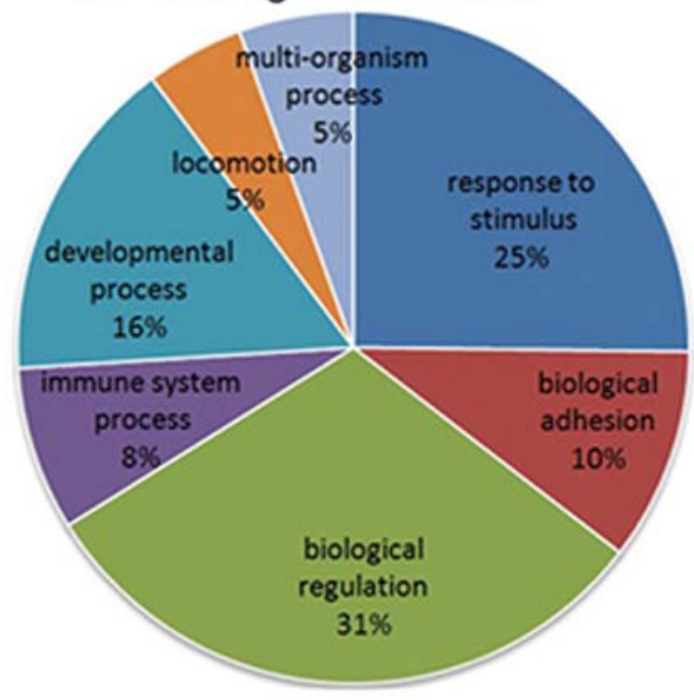

\section{B Molecular Function}

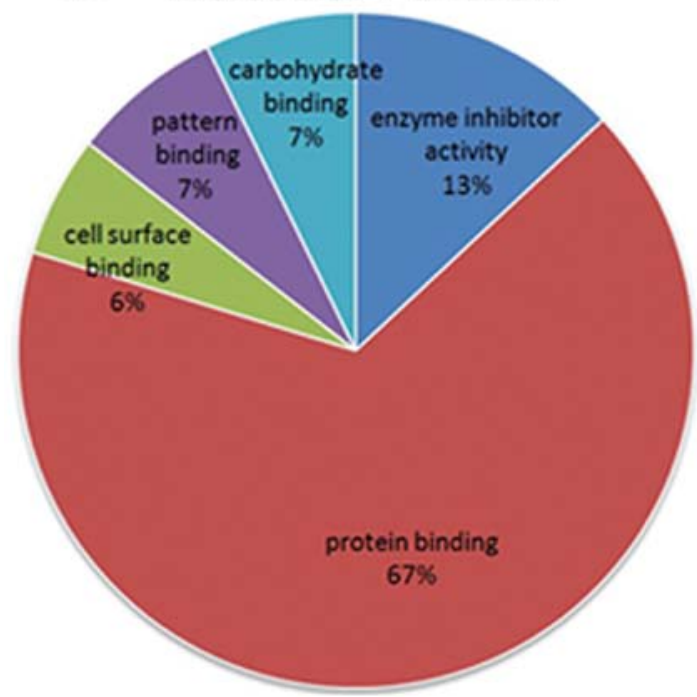

Figure 1. Gene Ontologies. The most significantly enriched (A) biological processes and (B) molecular functions according to DAVID Bioinformatics Resources based on the 111 differential proteins identified in TBM subjects.

Qualitative results. Data from the two MS repetitions identified 572 unique proteins according to the previously described parameter set. Only the 81 differential proteins with a \pm 1.2 -fold-change and $\mathrm{P}<0.05$ were selected for further analysis (Table II). The threshold criteria were selected based on literature investigating the reproducibility of iTRAQ ${ }^{\mathrm{TM}}$ quantification (23).

Annotation of differential proteins. Some differential proteins exhibited multiple functions and subcellular locations, which resulted in individual proteins being counted more than once. Considering that a given protein can be attributed to several functions and/or processes, we categorized differential proteins by their biological processes (Fig. 1A) and molecular functions (Fig. 1B) by pie chart. 'Response to stimulus', 'biological adhesion' and 'biological regulation' were the most over-represented biological processes. 'Enzyme inhibitor activity' was the most
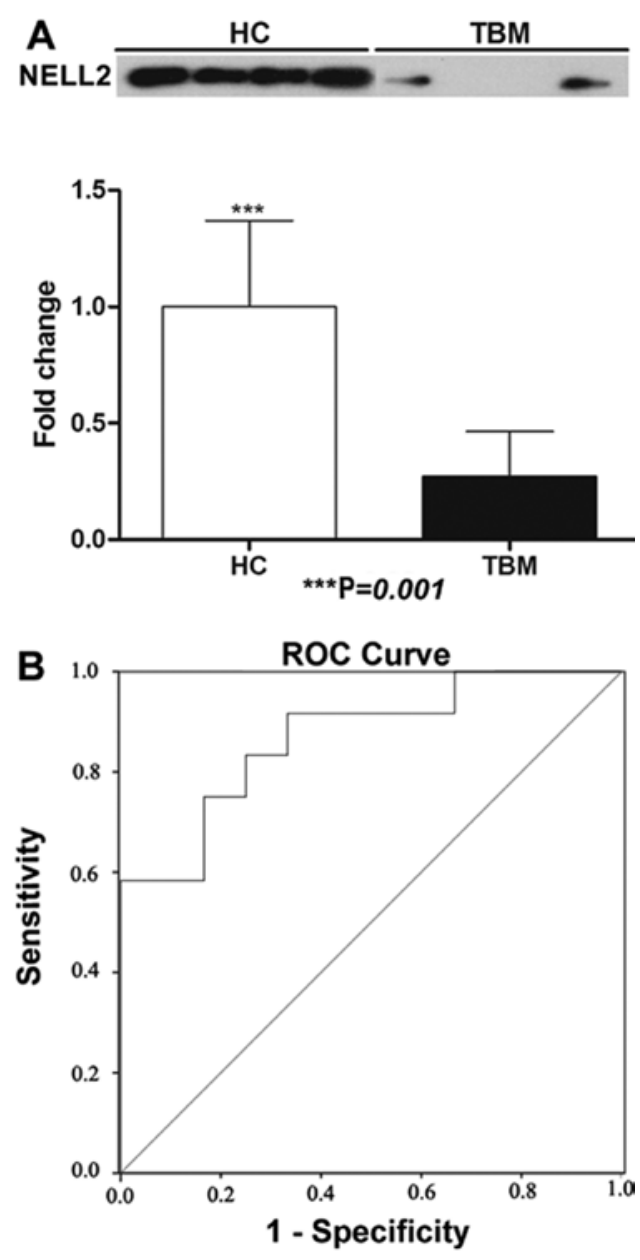

Figure 2. Western blot validation of NELL2. (A) NELL2 was significantly downregulated in tuberculous meningitis (TBM) subjects compared to healthy control (HC) subjects by western blotting (HC, $\mathrm{n}=12$; TBM, $\mathrm{n}=12$ ). (B) Receiver operating characteristic (ROC) analysis of NELL2 western blot data showing detection of TBM from HC with an AUC of 0.868, indicating that NELL2 in cerebrospinal fluid (CSF) shows promise as a diagnostic biomarker for TBM.

over-represented molecular function, distantly followed by 'protein binding' and 'cell surface binding'.

Altered biological pathways by KEGG. The 81 differential proteins were analyzed for KEGG over-representation of pathways ('proteomic phenotyping') (24) to obtain insights into the functional differences between the CSF proteomes of TBM and $\mathrm{HC}$ subjects. The top three-ranking canonical KEGG pathways are listed in Table II. Inflammatory processes were the most statistically over-represented, including complement and coagulation cascades ranking first $(\mathrm{P}<0.001)$ and cell adhesion molecules $(\mathrm{CAMs})$ ranking third $(\mathrm{P}<0.01)$.

Validation of NELL2 by western blotting. The neuron-specific differential protein with the largest absolute fold-change value, neural epidermal growth factor-like like 2 (NELL2), was selected for western blot validation (Table II). In accordance with iTRAQ ${ }^{\mathrm{TM}}$, NELL2 decreased significantly in TBM compared to HC subjects (Fig. 2A). ROC curves were constructed using the western blotting data, in which the AUC, sensitivity, and specificity were $0.868,83.3$ and $75 \%$, respectively (Fig. 2 B). 
Table III. Top ten-ranking canonical KEGG pathways associated with differential proteins.

\begin{tabular}{lcccc}
\hline & \multicolumn{3}{c}{$\begin{array}{c}\text { No. of } \\
\text { molecules }\end{array}$} & \\
\cline { 2 - 3 } KEGG pathway & Mapping & $\%$ & P-value \\
\hline $\begin{array}{l}\text { Complement and coagulation } \\
\text { cascades }\end{array}$ & 10 & 16.7 & $2.40 \mathrm{e}^{-11}$ \\
$\begin{array}{l}\text { Prior diseases } \\
\text { Cell adhesion molecules (CAMs) }\end{array}$ & 4 & 6.7 & $7.00 \mathrm{e}^{-4}$ \\
\hline
\end{tabular}

\section{Discussion}

In the present study, using an iTR $\mathrm{AQ}^{\mathrm{TM}}$-based quantitative proteomic approach, we compared 12 CSF samples from TBM subjects to $12 \mathrm{CSF}$ samples from demographically-matched $\mathrm{HC}$ subjects. Among the 81 differential proteins identified, the neuron-specific protein with the largest absolute fold-change value, NELL2, was selected for western blotting validation and was found to be significantly decreased in TBM subjects compared to HC subjects. NELL2 had $83.3 \%$ sensitivity and $75 \%$ specificity and shows promise as a diagnostic biomarker for TBM.

Inflammation. KEGG analysis revealed over-representation in two inflammation-associated processes, namely complement and coagulation cascades and CAMs (Table III). As MTB infects and persists inside host macrophages, MTB infection is naturally accompanied by a host inflammatory response (25). In the initial stage, MTB activates several pro-inflammatory cytokines, including tumor necrosis factor- $\alpha($ TNF- $\alpha)$ (26), interleukin 1 (IL-1) (27), and reactive oxygen/nitrogen intermediaries (ROI/RNI) that promote MTB termination (28). Simultaneously, MTB induces the release of anti-inflammatory molecules, such as transforming growth factor- $\beta$ (TGF- $\beta$ ) (29) and IL-10 (30), to suppress the pro-inflammatory response and maintain MTB survival. In $\sim 90 \%$ of MTB cases, the host inflammatory response results in granuloma formation, which is sufficient to contain the infection and prevent disease. However, in $5-10 \%$ of MTB cases, the pathogen evades or subverts the host's granulomatous inflammatory response, producing either a latent or active infection (31). Therefore, the outcome of MTB infection depends on a complex set of host-pathogen interactions, and further investigation into the differential proteins associated with the host inflammatory response can offer insight into the pathogenesis and treatment of TBM. For example, drugs that intervene in the host inflammatory response, such as TGF- $\beta$ blockers, have been suggested as a method to prevent the spread of MTB infection (32).

Glycolysis/gluconeogenesis. Two significantly altered proteins including neogenin and fructose-bisphosphate aldolase related to glycolysis/gluconeogenesis were identified. MTB prefers to utilize host fatty acids as an energy source. Thus, glycolytic/gluconeogenic proteins that are involved in fatty acid utilization would be required for the growth and persistence of MTB in vivo (33).

NELL2. TBM is characterized by meningeal inflammation, CSF flow obstruction, fibrogelatinous exudates, and arterial vasculitis that traverse the exudates, resulting in cerebral infarction $(34,35)$. TBM sometimes results in tuberculoma formation (36), which is most commonly observed in the cerebellum, cerebrum and pons (37). In the present study, by magnetic resonance imaging (MRI), the 12 TBM subjects excibited basal meningeal enhancement consistent with TBM, seven of whom excibited cerebral infarction shown by high DWI signal intensity. These findings suggest a strong possibility of brain neuronal cell damage via vasculitis and tuberculoma formation. Considering that CSF neuron-specific enolase (NSE) is the most commonly applied biomarker of nervous tissue damage and can act as an early diagnostic marker of TBM $(38,39)$, we selected NELL2 for western blotting validation due to its neuronal specificity and high absolute fold-change value. NELL2, also known as neural epidermal growth factor-like like 2, NEL-like protein 2, Nel-related protein 2 (NRP2), and protein kinase C-binding protein, is a cytoplasmic enzyme that is expressed almost exclusively in the nervous system (40), predominantly in neurons with the highest density in the hippocampus and cerebral cortex (41). NELL2 promotes neuronal differentiation and survival during embryonic development (42) through mitogen-activated protein kinases (MAPK) (43), stimulates motor and sensory neuron differentiation in dorsal root ganglia (44), and promotes the differentiation of neural progenitor cells into neurons (45). NELL2 knock-out mice have demonstrated learning impairment in the Morris water maze task.

The present study revealed significantly decreased levels of NELL2 in TBM patients. As neuron-produced NELL2 supports neuronal survival and differentiation and neurons are compromised in TBM, it is reasonable to surmise that the reduced NELL2 levels observed in TBM patients result from brain neuronal cell damage or death. Additional study is required to determine whether there is a TBM disease stage in which NELL2 significantly increases to compensate for brain neuronal cell damage.

There are several limitations to the present study. First, eight of the $12 \mathrm{TBM}$ patients also had pulmonary TB, and another TBM patient had vertebral TB. As $25 \%$ of miliary TB patients also have TBM, presumably by transmission across the blood-brain barrier (46), future studies should include TBM patient cohorts with and without pulmonary TB, which may provide additional novel biomarkers of TBM. Second, the World Health Organization (WHO) recommends that all newly diagnosed human immunodeficiency virus (HIV) and syphilis patients should be tested for TB, as individuals with HIV or syphilis and latent TB infection are at a significantly higher risk of developing active TB (47). However, since no subjects of the present study were co-infected with HIV or syphilis, future studies should include cohorts of HIV or syphilis co-infected patients to improve our understanding of MTB host interaction in these patient populations. Third, the influence of anti-TB agents on differential protein expression was not assessed and should be taken into consideration in future studies. Fourth, in order to improve specificity for 
TBM, the current findings require further validation in larger sample sets that include other intracranial infectious diseases.

In conclusion, an iTRAQ ${ }^{\mathrm{TM}}$-based quantitative proteomic approach was utilized in the present study to identify differential proteins in the CSF of TBM patients and healthy controls. A total of 111 differential proteins were identified, and NELL2 was validated by western blotting as a promising diagnostic biomarker for TBM. Further evaluation of these findings in larger studies including medicated and unmedicated cohorts with other intracranial infectious diseases is required for clinical translation.

\section{Acknowledgements}

We graciously thank Dr Wei Ding (Shanghai Institute for Biological Sciences, Chinese Academy of Sciences) for his assistance with the mass spectrometric analysis and his technical assistance with the KEGG database. We also extend thanks to Dr N.D. Melgiri for editing and proofreading the manuscript. The present study was supported by the National Key Scientific Program of China (nos. 2009CB918300 and 2012CB910602) and the National Nature Science Foundation of China (no. 81371310).

\section{References}

1. WHO global tuberculosis control report 2010. Summary. Cent Eur J Public Health 18: 237, 2010.

2. Corbett EL, Watt CJ, Walker N, Maher D, Williams BG and Raviglione MC: The growing burden of tuberculosis: global trends and interactions with the HIV epidemic. Arch Intern Med 163: 1009-1021, 2003.

3. Zhao Y, Xu S, Wang L, Chin DP, Wang S and Jiang G: National survey of drug-resistant tuberculosis in China. N Engl J Med 366 2161-2170, 2012

4. Thwaites GE: Advances in the diagnosis and treatment of tuberculous meningitis. Curr Opin Neurol 26: 295-300, 2013

5. Thwaites GE, van Toorn R and Schoeman J: Tuberculous meningitis: more questions, still too few answers. Lancet Neurol 12: 999-1010, 2013.

6. Rylance J, Pai M, Lienhardt C and Garner P: Priorities for tuberculosis research: a systematic review. Lancet Infect Dis 10: 886-892, 2010

7. McNerney R, Maeurer M, Abubakar I, et al: Tuberculosis diagnostics and biomarkers: needs, challenges, recent advances, and opportunities. J Infect Dis 205: S147-S158, 2012.

8. Yew WW, Borgdorff MW and Enarson DA: New tools for global tuberculosis control: are we any closer? Int J Tuberc Lung Dis 16 : 855-856, 2012.

9. Thwaites G, Chau TT, Mai NT, Drobniewski F, McAdam K and Farrar J: Tuberculous meningitis. J Neurol Neurosurg Psychiatry 68: 289-299, 2000.

10. Eintracht S, Silber E, Sonnenberg P, Koornhof HJ and Saffer D: Analysis of adenosine deaminase isoenzyme-2 (ADA $)_{2}$ in cerebrospinal fluid in the diagnosis of tuberculosis meningitis. J Neurol Neurosurg Psychiatry 69: 137-138, 2000.

11. Donald PR, Malan C, van der Walt A and Schoeman JF: The simultaneous determination of cerebrospinal fluid and plasma adenosine deaminase activity as a diagnostic aid in tuberculous meningitis. S Afr Med J 69: 505-507, 1986.

12. Mishra OP, Loiwal V, Ali Z, Nath G and Chandra L: Cerebrospinal fluid adenosine deaminase activity for the diagnosis of tuberculous meningitis in children. J Trop Pediatr 42: 129-132, 1996

13. Mudaliar AV, Kashyap RS, Purohit HJ, Taori GM and Daginawala HF: Detection of $65 \mathrm{kD}$ heat shock protein in cerebrospinal fluid of tuberculous meningitis patients. BMC Neurol 6: 34, 2006.

14. Donald PR and Malan C: Cerebrospinal fluid lactate and lactate dehydrogenase levels as diagnostic aids in tuberculous meningitis. S Afr Med J 67: 19-20, 1985.
15. Kataria J, Rukmangadachar LA, Hariprasad G, O J, Tripathi M and Srinivasan A: Two dimensional difference gel electrophoresis analysis of cerebrospinal fluid in tuberculous meningitis patients. J Proteomics 74: 2194-2203, 2011.

16. Walzl G, Ronacher K, Hanekom W, Scriba TJ and Zumla A Immunological biomarkers of tuberculosis. Nat Rev Immunol 11: 343-354, 2011.

17. Waybright TJ: Preparation of human cerebrospinal fluid for proteomics biomarker analysis. Methods Mol Biol 1002: 61-70, 2013.

18. Kumar GS, Venugopal AK, Mahadevan A, Renuse S Harsha HC and Sahasrabuddhe NA: Quantitative proteomics for identifying biomarkers for tuberculous meningitis. Clin Proteomics 9: 12, 2012.

19. Tumani H, Lehmensiek V, Lehnert S, Otto M and Brettschneider J: 2D DIGE of the cerebrospinal fluid proteome in neurological diseases. Expert Rev Proteomics 7: 29-38, 2010.

20. Ross PL, Huang YN, Marchese JN, Williamson B, Parker K, Hattan S, et al: Multiplexed protein quantitation in Saccharomyces cerevisiae using amine-reactive isobaric tagging reagents. Mol Cell Proteomics 3: 1154-1169, 2004.

21. Ahuja GK, Mohan KK, Prasad K and Behari M: Diagnostic criteria for tuberculous meningitis and their validation. Tuber Lung Dis 75: 149-152, 1994.

22. Dennis G Jr, Sherman BT, Hosack DA, Yang J, Gao W and Lane HC: DAVID: Database for Annotation, Visualization, and Integrated Discovery. Genome Biol 4: P3, 2003.

23. Brea D, Sobrino T, Blanco M, Fraga M, Agulla $J$ and Rodriguez-Yanez M: Usefulness of haptoglobin and serum amyloid A proteins as biomarkers for atherothrombotic ischemic stroke diagnosis confirmation. Atherosclerosis 205: 561-567, 2009.

24. Pan C, Kumar C, Bohl S, Klingmueller U and Mann M: Comparative proteomic phenotyping of cell lines and primary cells to assess preservation of cell type-specific functions. Mol Cell Proteomics 8: 443-450, 2009.

25. Toossi Z: The inflammatory response in Mycobacterium tuberculosis infection. Arch Immunol Ther Exp (Warsz) 48: 513-519, 2000.

26. Valone SE, Rich EA, Wallis RS and Ellner JJ: Expression of tumor necrosis factor in vitro by human mononuclear phagocytes stimulated with whole Mycobacterium bovis BCG and mycobacterial antigens. Infect Immun 56: 3313-3315, 1988.

27. Wallis RS, Fujiwara $\mathrm{H}$ and Ellner JJ: Direct stimulation of monocyte release of interleukin 1 by mycobacterial protein antigens. J Immunol 136: 193-196,1986.

28. Bulut Y, Michelsen KS, Hayrapetian L, Naiki Y, Spallek R and Singh M: Mycobacterium tuberculosis heat shock proteins use diverse Toll-like receptor pathways to activate pro-inflammatory signals. J Biol Chem 280: 20961-20967, 2005

29. Hirsch CS, Toossi Z, Othieno C, Johnson JL, Schwander SK and Robertson S: Depressed T-cell interferon- $\gamma$ responses in pulmonary tuberculosis: analysis of underlying mechanisms and modulation with therapy. J Infect Dis 180: 2069-2073, 1999.

30. Lin Y, Zhang M, Hofman FM, Gong J and Barnes PF: Absence of a prominent Th 2 cytokine response in human tuberculosis. Infect Immun 64: 1351-1356, 1996.

31. Stavrum R, Stavrum AK, Valvatne H, Riley LW, Ulvestad E, Jonassen I, et al: Modulation of transcriptional and inflammatory responses in murine macrophages by the Mycobacterium tuberculosis mammalian cell entry (Mce) 1 complex. PLoS One 6: e26295, 2011.

32. Sasindran SJ and Torrelles JB: Mycobacterium tuberculosis infection and inflammation: what is beneficial for the host and for the hacterium? Front Microbiol 2: 2, 2011.

33. Shi L, Sohaskey CD, Pfeiffer C, Datta P, Parks M and McFadden J: Carbon flux rerouting during Mycobacterium tuberculosis growth arrest. Mol Microbiol 78: 1199-1215, 2010.

34. Thwaites GE and Schoeman JF: Update on tuberculosis of the central nervous system: pathogenesis, diagnosis, and treatment. Clin Chest Med 30: 745-754, 2009.

35. Malhotra HS, Garg RK, Singh MK, Agarwal A and Verma R: Corticosteroids (dexamethasone versus intravenous methylprednisolone) in patients with tuberculous meningitis. Ann Trop Med Parasitol 103: 625-634, 2009.

36. Garg RK: Tuberculosis of the central nervous system. Postgrad Med J 75: 133-140, 1999.

37. Damergis JA, Leftwich EI, Curtin JA and Witorsch P: Tuberculoma of the brain. JAMA 239: 413-415, 1978. 
38. Rodriguez-Nunez A, Cid E, Rodriguez-Garcia J, Camina F, Rodriguez-Segade S and Castro-Gago M: Neuron-specific enolase, nucleotides, nucleosides, purine bases, oxypurines and uric acid concentrations in cerebrospinal fluid of children with meningitis. Brain Dev 25: 102-106, 2003.

39. Song TJ, Choi YC, Lee KY and Kim WJ: Serum and cerebrospinal fluid neuron-specific enolase for diagnosis of tuberculous meningitis. Yonsei Med J 53: 1068-1072, 2012.

40. Watanabe TK, Katagiri T, Suzuki M, Shimizu F, Fujiwara T and Kanemoto N: Cloning and characterization of two nove human cDNAs (NELL1 and NELL2) encoding proteins with six EGF-like repeats. Genomics 38: 273-276, 1996.

41. Oyasu M, Kuroda S, Nakashita M, Fujimiya M, Kikkawa U and Saito N: Immunocytochemical localization of a neuron-specific thrombospondin-1-like protein, NELL2: light and electron microscopic studies in the rat brain. Brain Res Mol Brain Res 76: $151-160,2000$

42. Tong ZB, Bondy CA, Zhou J and Nelson LM: A human homologue of mouse Mater, a maternal effect gene essential for early embryonic development. Hum Reprod 17: 903-911, 2002.

43. Aihara K, Kuroda S, Kanayama N, Matsuyama S, Tanizawa K and Horie M: A neuron-specific EGF family protein, NELL2, promotes survival of neurons through mitogen-activated protein kinases. Brain Res Mol Brain Res 116: 86-93, 2003.
44. Nelson BR, Claes K, Todd V, Chaverra M and Lefcort F: NELL2 promotes motor and sensory neuron differentiation and stimulates mitogenesis in DRG in vivo. Dev Biol 270: 322-335, 2004.

45. Hwangpo TA, Jordan JD, Premsrirut PK, Jayamaran G, Licht JD and Iyengar R: G Protein-regulated inducer of neurite outgrowth (GRIN) modulates Sprouty protein repression of mitogen-activated protein kinase (MAPK) activation by growth factor stimulation. J Biol Chem 287: 13674-13685, 2012.

46. Jain SK, Paul-Satyaseela M, Lamichhane G, Kim KS and Bishai WR: Mycobacterium tuberculosis invasion and traversal across an in vitro human blood-brain barrier as a pathogenic mechanism for central nervous system tuberculosis. J Infect Dis 193: 1287-1295, 2006.

47. Goswami ND, Hecker EJ, Vickery C, Ahearn MA, Cox GM and Holl DP: Geographic information system-based screening for TB, HIV, and syphilis (GIS-THIS): a cross-sectional study. PLoS One 7: e46029, 2012. 